\title{
Uncommon Infection of an Epidermoid Cyst in the Face with Peptoniphilus harei - A Systematic Review of the Literature Regarding Treatment Strategy
}

\author{
Abazi S, Harnik B, Wiesli MG, Leiggener CS and \\ Augello M* \\ Department of Oral and Maxillofacial Surgery, \\ Kantonsspital Aarau, Switzerland \\ *Correspondling author: Augello M, Department of \\ Oral and Maxillofacial Surgery, Kantonsspital Aarau, \\ Switzerland
}

Received: November 08, 2018; Accepted: December 13, 2018; Published: December 20, 2018

\begin{abstract}
Purpose: Infections with Peptoniphilus harei are rare and no consens about treatment exists. Based on a case report, we report on our approach and make a review of the literature. To our knowledge, we are the first to be confronted with an infected epidermoid cyst in the facial area due to Peptoniphilus harei in its pure form.
\end{abstract}

Material and Methods: A smear test for microbiological examination and for culture determination was taken intraoperative. Peptoniphilus harei could be detected by using the matrix-assisted laser desorption ionization time-off light mass spectrometry (MOLDI-TOF) method. A literature research showed only few documentations of an infection with this microorganism.

Results: The post-operative course was uneventful. No antibiotics were needed. The microbiological examination detected Peptoniphilus harei as the only microorganism.

Conclusion: The clinical impact of Peptinophilus harei is not clearly clarified due to poor data. Resistance cannot currently be considered as a problem. In our case report compared to the other case reports, no antibiotic therapy was initiated. For the detection of the causative microorganism, a microbiological sample is recommended.

Keywords: Peptoniphilus harei; Peptostreptococcus harei; Epidermoidcyst

\section{Introduction}

Peptostreptococcus harei was first isolated from antral lavage, from the peritoneal cavity and from a suppurative sacral ulcer. Furthermore also from abscesses of the neck area [1]. The bacterium Peptostreptococcus was reclassified in 2001 to the genus Peptoniphilus, which also includes Peptoniphilus harei [2]. Peptoniphilus harei are non-spore-forming, gram-positive, obligate Anaerobic cocci. Peotinophilus harei has been found mainly in polymicrobial infections $[3,4]$. Only in a few cases, Peptinophilus harei has been isolated as the only pathogen without the involvement of other bacteria out of an infection [5-8].

In this case, report we document the first case of an infected epidermoid cyst of the face caused by Peptinophilus harei. To our knowledge, we are the first to be confronted with an infected epidermoid cyst in the facial area due to Peptoniphilus harei in its pure form.

\section{Case Report}

A 57-year-old man introduced himself to our department. $\mathrm{He}$ reported that 3 weeks before a pustula on the right he manipulated cheek. After that the patient observed a slowly rising swelling on the right cheek. In the course, it came to swelling with redness and painfull palpation of the soft tissues. Finally, with abscess formation in the right cheek the patient presented himself to our clinic. The patient was in good general condition with no history of any other diseases and no history of drugs administration. In addition, the social and sexual anamnes is showed no abnormalities. The patient was afebrile and presented no ague. Because of the clear clinical findings and good general condition without further diseases, we renounced a radiological and an examination of inflammation parameters. There was no indication to take blood cultures. On the day of the presentation the abscess incision with drainage was performed. A smear test for microbiological examination and for culture determination was taken intraoperatively. After 48 hours of cultivation, gram-positive cocci were found. The detection of Peptoniphilus harei was achieved using the matrix-assisted laser desorption ionization time-off light mass spectrometry (MOLDI-TOF) method. The result showed Peptoniphilus harei as the only microorganism. After another 24 hours, an antibiogram profile was made. The microorganism was sensitive to all the antibiotics we tested: penicillin, amoxicillin-clavulanic acid, ampicillin, clarithromycin, clindamycin and metronidazole. We did not initiate antibiotic therapy. With regular irrigation, there was a clear clinical improvement (Figure 1). During the therapy, the drainage could be removed. One week after abscess incision surgical excision of the tumorous tissue could be fulfilled (Figure 2). The histological examination revealed an epidermoid cyst without evidence of malignancy. The further course was uneventful. We could conclude the treatment two weeks after abscess incision.
Austin J Surg - Volume 5 Issue 9 - 2018

ISSN : 2381-9030 | www.austinpublishing group.com

Augello et al. () All rights are reserved
Citation: Abazi S, Harnik B, Wiesli MG, Leiggener CS and Augello M. Uncommon Infection of an Epidermoid Cyst in the Face with Peptoniphilus harei - A Systematic Review of the Literature Regarding Treatment Strategy. Austin J Surg. 2018; 5(9): 1155 


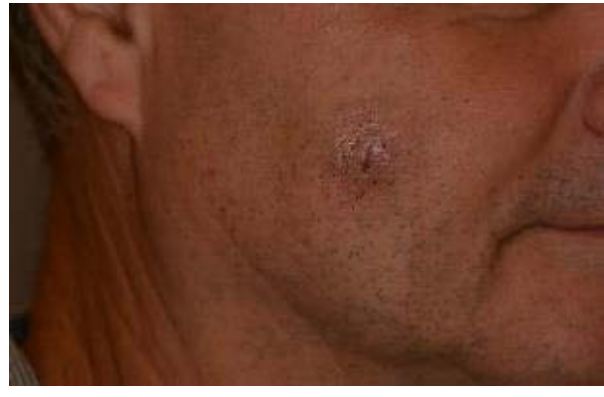

Figure 1: Clear clinical improvement.

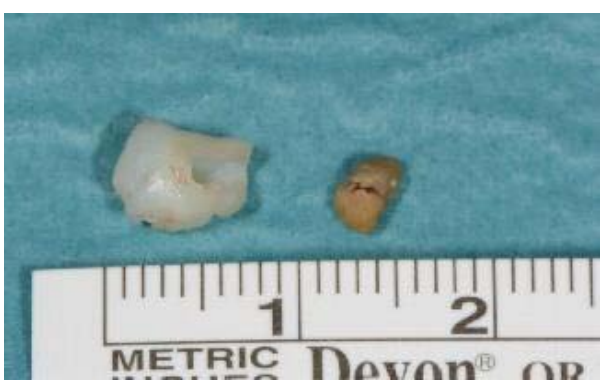

Figure 2: Surgical excision of the tumorous tissue.

\section{Systematic Review}

We aimed to identify all publications pertaining to infection with Peptinophilus harei in pure culture. We used the the Preferred Reporting Items for Systematic Reviews and Meta- Analyses (PRISMA). Published studies were found by utilizing a thorough search strategy of the PubMed, Google Scholar and Hand search. The following search terms were used: Peptoniphilus harei, Peptostreptococcus harei, epidermoid cyst. We used the chosen articles to identify more articles that are relevant. Eligibility criterion for inclusion in the review was the detection of Peptoniphilus harei in pure culture.

\section{Results and Discussion}

A systematic, multilingual review of medical literature showed that only in few cases Peptinophilus harei was isolated in pure culture. We examined 21 articles and identified five papers relevant to our research topic that met our criteria. The search strategy and flow diagram (Figure 3) are presented using the PRISMA guidelines.

The treatment of choice for infections caused by Peptinophilus harei is not established due to the lack of data. Ganiy Opeyemi Abdulrahman G.O, and Christopher Alan Gateley C.A, isolated Peptinophilus harei together with A. turicensis from a breast abscess after piercing. Cobo et al. isolated Peptinophilus harei from a purulent peritoneal inflammation in a patient with bowel obstruction in April 2016 and recently in a 60-year-old man from an infected lymphocele after radical prostatectomy. In all three cases, surgical assessment and systemic antibiotic therapy were performed [5-7]. In our case, antibiotic therapy was not required. Murdoch et al. found two strains in pure culture from abscesses in different locations. However, the therapy was not described and no statement can be made here regarding the clinical significance [1].
Peptoniphilus harei belongs to the Gram-Positive Anaerobic cocci (GPAC). The genus Peptoniphilus was originally described as Peptococcus, which was modified to Peptostreptococcus in 1986 [5]. In July 1997 these Peptostreptococci were reported by Murdoch et al. investigated further with phenotypic and genotypic methods and new subtype was isolated for the first time from antral lavage, the peritoneal cavity and a suppurative sacral ulcer. This new species of the genus Peptostreptococcus was then classified as Peptoniphilus harei [1]. The genus Peptostreptococcus itself was reclassified in 2001 to the genus Peptoniphilus [2]. Peptinophilus harei occurs mainly in polymicrobial infections $[3,4]$.

So far, only a few have been isolated in pure culture. Two strains were detected in pure culture from abscesses [1]. In a 22-year-old woman, Peptinophilus harei was isolated together with A. turicensis from a breast abscess after piercing. However, no statement can be made here regarding the clinical significance [6]. Cobo et al. have identified Peptinophilus harei from a purulent peritoneal inflammation in a patient with bowel obstruction in April 2016 and recently in a 60-year-old man from an infected lymphocele after radical prostatectomy. In both cases, the source of infection was unknown. Because Peptinophilus harei belongs to the skin flora, it is assumed that the skin was responsible as the path of contamination $[5,7]$. We assume that our patient's skin also served as the path of contamination. Our case report describes for the first time the unique isolation of Peptinophilus harei from an epidermoid cyst in the facial area. Compared to our case where the infection arosed spontaneously, Cobo's et al. datum case report was preceded by surgery. Also in the second case report of Cobo et al. a patient suffering from a bilateral ovarian carcinoma was operated several times. In the described laparotomy the microbiological examination of the ascites fluid was arranged, in which the bacterium Peptinophilus harei was identified [5]. It is therefore unclear whether the infection was due to one of the pre-surgery or by the immunosuppressive underlying disease.

The detection of Peptoniphilus harei infection passed via the establishment of a culture. Strain identification is usually done by phenotypic tests. The difficulty here is the phenotypic similarity and as well as the comparable biochemical properties of Peptoniphilus harei and Peptoniphilus asaccharolyticus. For this reason, more specific identification using molecular biological methods such as $16 \mathrm{~S}$ rRNA sequencing is of great importance. The recent technology of MatrixAssisted Laser Mass Spectrometry (MALDI-TOF) for the routine identification of bacteria, especially anaerobes, allows cost-effective identification of clinical isolates with high accuracy and speed $[9,10]$. In MALDI-TOF, the analysis molecule is added to a saturated, lowmass organic compound, also called matrix, while crystals form (cocrystallization). The analysis matrix-crystals, which are located on a metal plate, are excited with a UV laser beam with a wavelength of $337 \mathrm{~nm}$ for a short time, so that no damage to the analysis molecule arises. Photons of the laser beam lead to sublimation and ionization of the sample to be examined. The analysis molecules and the matrix are placed into the mass spectrometer and mass spectrometry is performed [11]. To analyze the pathogens present in our smear test, we applied the system of the MALDI-TOF due to the many advantages.

An antibiotic treatment of choice for infections caused by 


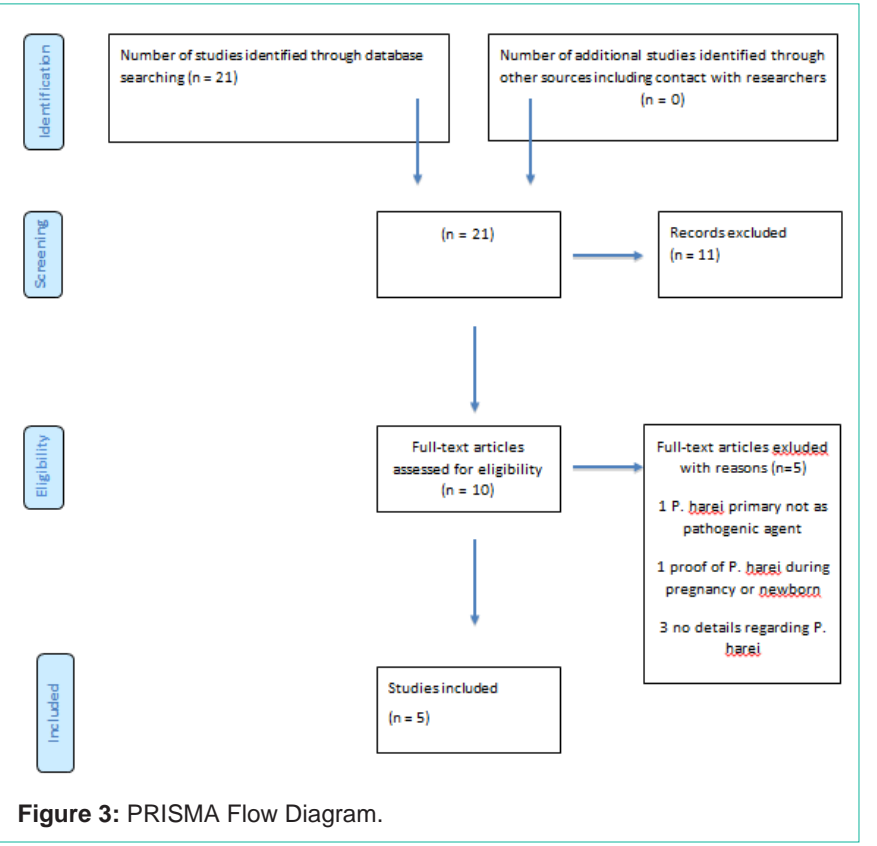

Peptinophilus harei is not established due to the lack of data. Jeverica et al. demonstrated that Peptoniphilus harei was resistant to penicillin in $1 \%$ of cases and resistant to clindamycin even in $29 \%$ of cases. In contrast, in $0 \%$ of cases they were resistant to amoxicillin-clavulanic acid, imipenem and metronidazole [12]. Cobo et al. reported in their case a high minimal inhibitory concentration for metronidazole and can be considered resistant according to the European Committee on Antimicrobial Susceptibility Testing (EUCAST) criteria [5]. In our case, Peptoniphilus harei was sensitive to all the antibiotics we tested. In view of the overall situation, the resistance of Peptoniphilus harei cannot currently be considered as a problem. In our case, antibiotic therapy was not required. Therefore, it can be assumed that after a complete surgical removal of the infected tissue, in the absence of general symptoms, and no presence of immune defiency an antibiotic therapy is not mandatory.

Skin infections are most commonly caused by group A betahemolytic streptococci and staphylococcus aureus [13]. Furthermore, aerobic and anerobic pathogens often coexist in acute and chronic infections, as they benefit from a synergistic effect [3]. In our case, Peptoniphilus harei caused a cheek abscess without involvement of other bacteria and as the only pathogenic agent.

\section{Conclusion}

The clinical impact of Peptinophilus harei is not clearly clarified due to poor data. Resistance cannot currently be considered as a problem. In our case report compared to the other case reports, no antibiotic therapy was initiated. For the detection of the causative microorganism, a microbiological sample is recommended.

\section{Consent}

Written informed consent was obtained from the patient for publication of this case report and accompanying images. A copy of the written consent is available for review by the Editor-in-Chief of this journal on request.

\section{References}

1. Murdoch DA, Collins A, Willems JM, Hardie KA, Young JT. Magee, Description of three new species of the genus Peptostreptococcus from human clinical specimens: Peptostreptococcus harei $\mathrm{sp}$ nov, Peptostreptococcus ivorii $\mathrm{sp}$ nov, and Peptostreptococcus octavius sp Nov. Int J Syst Bacteriol. 1997; 47: e781-e787

2. Ezaki T, Kawamura Y, Li N, Li ZY, Zhao L, Shu S. Proposal of the genera Anaerococcus gen. nov., Peptoniphilus gen. nov and Gallicola gen. nov. Formembers of the genus Peptostreptococcus. Int J Syst Evol Microbiol. 2001; 51: 1521-1528.

3. Rahim K, Saleha S, Zhu X, Huo L, Basit A, Franco OL. Bacterial Contribution in Chronicity of Wounds. Microb Ecol. 2017; 73: 710-721.

4. Dowd SE, Wolcott RD, Sun Y, McKeehan T, Smith E, Rhoads D. Polymicrobia nature of chronic diabetic foot ulcer biofilm infections determined using bacterial tag encoded FLX amplicon pyrosequencing (bTEFAP). PLoS One. 2008; 3: e3326.

5. Cobo F, Rodríguez-Granger J, Sampedro A, Navarro-Marí JM. Peritoneal infection due to Peptoniphilus harei in a patient with intestinal occlusion. Anaerobe. 2017; 44: 126-127.

6. Abdulrahman GO Jr, Gateley CA. Primary actinomycosis of the breast caused by Actinomyces turicensis with associated Peptoniphilus harei. Breast Dis. 2015; 35: 45-47.

7. Cobo F. Lymphocele infection due to Peptoniphilus harei after radical prostatectomy. Med Mal Infect. 2018; 48: 154-155.

8. Murdoch DA, Mitchelmore IJ, Tabaqchali S. The clinical importance of grampositive anaerobic cocci isolated at St Bartholomew's Hospital, London, in 1987. J Med Microbiol. 1994; 41: 36-44.

9. La Scola B, Fournier PE, Raoult D. Burden of emerging anaerobes in the MALDI-TOF and 16S rRNA gene sequencing era. Anaerobe. 2011; 17: 106112.

10. Shilnikova II, Dmitrieva NV. Evaluation of Antibiotic Susceptibility of GramPositive Anaerobic cocci Isolated from Cancer Patients of the NN. Blokhin Russian Cancer Research Center. J Pathog. 2015; 2015: 648134.

11. Clark AE, Kaleta EJ, Arora A, Wolk DM. Matrix-assisted laser desorption ionization-time of flight mass spectrometry: a fundamental shift in the routine practice of clinical microbiology. Clin Microbiol Rev. 2013; 26: 547-603.

12. Jeverica S, Kolenc U, Mueller-Premru M, Papst L. Evaluation of the routineantimicrobial susceptibility testing results of clinically significant anaerobic bacteria in a Slovenian tertiary-care hospital in 2015. Anaerobe. 2017; 47: 64-69.

13. Brook I. The role of anaerobic bacteria in cutaneous and soft tissue abscesses and infected cysts. Anaerobe. 2007; 13: 171-177.
Austin J Surg - Volume 5 Issue 9 - 2018

ISSN : 2381-9030 | www.austinpublishing group.com

Augello et al. () All rights are reserved
Citation: Abazi S, Harnik B, Wiesli MG, Leiggener CS and Augello M. Uncommon Infection of an Epidermoid Cyst in the Face with Peptoniphilus harei - A Systematic Review of the Literature Regarding Treatment Strategy. Austin J Surg. 2018; 5(9): 1155. 